\title{
Unprovable Security of Perfect NIZK and Non-interactive Non-malleable Commitments
}

\author{
Rafael Pass ${ }^{\star}$ \\ Cornell University \\ rafael@cs.cornell.edu
}

\begin{abstract}
We present barriers to provable security of two fundamental (and well-studied) cryptographic primitives perfect non-interactive zero knowledge (NIZK), and non-malleable commitments:

- Black-box reductions cannot be used to demonstrate adaptive soundness (i.e., that soundness holds even if the statement to be proven is chosen as a function of the common reference string) of any statistical (and thus also perfect) NIZK for $\mathcal{N} \mathcal{P}$ based on any "standard" intractability assumptions.

- Black-box reductions cannot be used to demonstrate non-malleability of non-interactive, or even 2-message, commitment schemes based on any "standard" intractability assumptions.
\end{abstract}

We emphasize that the above separations apply even if the construction of the considered primitives makes a non-black-box use of the underlying assumption.

As an independent contribution, we suggest a taxonomy of gamebased intractability assumption based on 1) the security threshold, 2) the number of communication rounds in the security game, 3) the computational complexity of the game challenger, 4) the communication complexity of the challenger, and 5) the computational complexity of the security reduction.

\section{Introduction}

Modern Cryptography relies on the principle that cryptographic schemes are proven secure based on mathematically precise assumptions; these can be general - such as the existence of one-way functions - or specific - such as the hardness of factoring products of large primes. The security proof is a reduction

\footnotetext{
* Pass is supported in part by a Alfred P. Sloan Fellowship, Microsoft New Faculty Fellowship, NSF Award CNS-1217821, NSF CAREER Award CCF-0746990, NSF Award CCF-1214844, AFOSR YIP Award FA9550-10-1-0093, and DARPA and AFRL under contract FA8750-11-2- 0211. The views and conclusions contained in this document are those of the authors and should not be interpreted as representing the official policies, either expressed or implied, of the Defense Advanced Research Projects Agency or the US Government.
} 
that transforms any attacker $A$ of the scheme into a machine that breaks the underlying assumption (e.g., inverts an alleged one-way function). This study has been extremely successful, and during the past three decades many cryptographic tasks have been put under rigorous treatment and numerous constructions realizing these tasks have been proposed under a number of well-studied complexity-theoretic hardness assumptions.

We here consider two fundamental cryptographic primitives-perfect noninteractive zero-knowledge with adaptive statements and non-interactive nonmalleable commitments - for which security proofs based on well-studied intractability assumptions have remained elusive.

Perfect NIZK with Adaptive Inputs. A non-interactive zero-knowledge (NIZK) protocol [BFM88] is protocol between two parties, a Prover, and a Verifier, through which the Prover can non-interactively (i.e., by sending a single message $\pi$ ) convince the Verifier of the validity of a statement $x$, only if $x$ is true (this is called the soundness property), while at the same time revealing nothing beyond the fact that $x$ is true (this is called the zero-knowledge property). To make such constructs possible both parties are additionally assumed to have access to a "Common Reference String" (CRS) that has been ideally sampled according to some distribution. The original definition of [BFM88] only considered non-adaptive notions of soundness and zero-knowledge: Roughly speaking, the (non-adaptive) soundness condition requires that for every false statement $x \notin L$, with high probability over the choice of the CRS, any proof $\pi$ output by a malicious prover will be rejected by the verifier. The (non-adaptive) zeroknowledge property, on the other hand, requires that for every true statement $x \in L$, the joint distribution consisting of the reference string, and an honestly generated proof, can be reconstructed by a simulator. In both of these properties, the statement $x$ is required to be fixed before the reference string is known. Feige, Lapidot and Shamir [FLS90] introduced stronger adaptive notions of both soundness and zero-knowledge; roughly speaking, here soundness and zero-knowledge should hold even if the statement $x$ is adversarially chosen as a function of the reference string.

As with traditional zero-knowledge protocols, NIZKs come in several flavors: computational NIZK, statistical NIZK, and perfect NIZK. In the computational notion, the simulator's output is only required to be computationally indistinguishable from an honestly generated view, whereas in the statistical (resp. perfect) variants, it is required to be statistically close (resp identical) to an honestly generated view. Computational NIZK with adaptive zero-knowledge and soundness were constructed early on based on standard cryptographic intractability assumptions [FLS90, BY96, but constructions of statistical and perfect NIZK were elusive.

Only recently, a breakthrough result by Groth, Ostrovsky and Sahai (GOS) GOS06 provided a construction of a perfect NIZK for $\mathcal{N} \mathcal{P}$ based on the hardness of a number theoretic assumption over bilinear groups. Their protocol satisfies the adaptive notion of zero-knowledge; however, it only satisfies the non-adaptive notion of soundness (that is, soundness is no longer guaranteed to hold if the 
attacker chooses a statement $x \notin L$ as a function of the common reference string). We here focus on whether there exists a perfect NIZK for $\mathcal{N} \mathcal{P}$ with both adaptive soundness and zero-knowledge.

A step towards answering this question appears in the work of Abe and Fehr AF07, which presented a perfect NIZK for $\mathcal{N} \mathcal{P}$ with both adaptive soundness and zero-knowledge, using an "knowledge-extractaction" assumption (simular to the "knowledge-of-exponent" assumption of Dam91]), as opposed to a computational-intractability assumption. Abe and Fehr also demonstrate that certain (arguably natural) types of proof techniques - which they refer to as "direct" black-box reductions - cannot be used to prove adaptive soundness of perfect NIZKs for $\mathcal{N} \mathcal{P}$. Their notion of a "direct" proof, however, is quite restrictive (very roughly speaking, it requires the security reduction to "directly embedd" some hard instance into the CRS in a "structure preserving way") 1

Non-interactive Non-malleable Commitments. Often described as the "digital" analogue of sealed envelopes, commitment schemes enable a sender to commit itself to a value while keeping it secret from the receiver. This property is called hiding. Furthermore, the commitment is binding, and thus in a later stage when the commitment is opened, it is guaranteed that the "opening" can yield only a single value determined in the committing stage. For many applications, however, the most basic security guarantees of commitments are not sufficient. For instance, the basic definition of commitments does not rule out an attack where an adversary, upon seeing a commitment to a specific value $v$, is able to commit to a related value (say, $v-1$ ), even though it does not know the actual value of $v$. This kind of attack might have devastating consequences if the underlying application relies on the independence of committed values (e.g., consider a case in which the commitment scheme is used for securely implementing a contract bidding mechanism). In order to address the above concerns, Dolev, Dwork and Naor introduced the concept of non-malleable commitments DDN00. Loosely speaking, a commitment scheme is said to be non-malleable if it is infeasible for an adversary to "maul" a commitment to a value $v$ into a commitment to a related value $\tilde{v}$.

More precisely, we consider a man-in-the-middle (MIM) attacker that participates in two concurrent executions of a commitment scheme $\Pi$; in the "left" execution it interacts with an honest committer; in the "right" execution it interacts with an honest receiver. Additionally, we assume that the players have $n$-bit identities (where $n$ is polynomially related to the security parameter), and that the commitment protocol depends only on the identity of the committer; we sometimes refer to this as the identity of the interaction. Intuitively, $\Pi$ being non-malleable means that if the identity of the right interaction is different

\footnotetext{
${ }^{1}$ Among other things, the structure preserving property requires that if the "hard instance" being directly embedded in the CRS is true, the CRS is valid, and if the hard instance is false, then the CRS is "invalid". This property can never hold when considering NIZK in the Uniform Reference String model (as every CRS is valid), and as such their result holds vacously when considering NIZK in the Uniform Reference String model.
} 
than the identity of the left interaction (i.e., $A$ does not use the same identity as the left committer), the value $A$ commits to on the right does not depend on the value it receives a commitment to on the left; this is formalized by requiring that for any two values $v_{1}, v_{2}$, the value $A$ commits to after receiving left commitments to $v_{1}$ or $v_{2}$ are indistinguishable.

The first non-malleable commitment protocol was constructed by Dolev, Dwork and Naor DDN00 in 1991. The security of their protocol relies on the minimal assumption of one-way functions and requires $\Omega(\log n)$ rounds of interaction, where $k \in N$ is the length of party identities. The round-complexity of non-malleable commitments has since been extensively studied (see e.g., Bar02, PR05b, PR05a, LPV08, LP09, PW10, Wee10], leading up to constant round protocols based on one-way functions [LP11, Goy11].

The question of whether non-interactive, or even 2-round, non-malleable commitments exist, however, is wide open. (We note that in the Common Reference String model, constructions of non-interactive non-malleable commitments are known CIO98; we here focus on constructions in the plain model, without any set-up.) Some initial progress towards this question can be found in PPV08 where a construction of non-interactive non-malleable commitments based on a new hardness assumption is given; this assumption, however, has a strong nonmalleability flavor; as such, it provides little insight into the question of whether non-malleability can be obtained from a "pure" hardness assumptions (such as e.g., the hardness of factoring).

\subsection{Our Results}

The main result of this paper is showing that Turing (i.e., black-box) reductions cannot be used to base the security of the above-mentioned primitives, on a general class of intractability assumption.

More precisely, following Naor [Nao03] (see also [DOP05, HH09, RV10, Pas11, GW11]), we model an intractability assumption as an arbitrary game between a (potentially) unbounded challenger $C$, and an attacker $A$. $A$ is said to break the assumption $C$ with respect to the threshold $t$ if it can make $C$ output 1 with probability non-negligibly higher than the threshold $t$. All traditional cryptographic hardness assumptions (e.g., the hardness of factoring, the hardness of the discrete logarithm problem, the decisional Diffie-Hellman problem etc.) can be modeled as 2-round challengers $C$ with the threshold $t$ being either 0 (in case of the factoring or discrete logarithm problems) or $1 / 2$ (in case of the decisional Diffie-Hellman problem). In all these examples $C$ is polynomial-time; Naor Nao03] and Gentry and Wichs [GW11] refer to such assumptions as "falsifiable". For generality, we here (following [Pas11]) refer to these as "efficient-challenger" assumptions. More generally, we refer to an assumption where the challenger can be implemented in time (resp. size) $T(\cdot)$ as a " $T(\cdot)$-time (resp. size) challenger assumption" Note that more "esoteric" assumptions such as the "one-more discrete logarithm assumption" BNPS03, BP02, or "adaptive one-way functions" PPV08, are not efficient-challenger assumptions, but they are exponential-time challenger assumptions. 
Our first result rules out basing statistical (and thus also perfect) NIZK with adaptive soundness on efficient-challenger (a.k.a falsifiable) assumptions.

Theorem 1 (Main Theorem 1-Informally stated). Assume the existence of (non-uniformly hard) one-way functions. Then there exists an $\mathcal{N} \mathcal{P}$-language $L$ such that the following holds. Let $\Pi$ be a statistical non-interactive adaptively zero-knowledge argument for L. Assume there exists a polynomial-time Turing reduction $R$ such that $R^{A}$ breaks the efficient-challenger assumption $C$ w.r.t. the threshold $t$ for every $A$ that breaks adaptive soundness of $\Pi$. Then $C$ can be broken in polynomial-time with respect to the threshold $t$.

We next show that if we additionally assume the existence of sub-exponential one-way functions, and consider the constructions of NIZK for proving any polynomial-length (in the security parameter) statement in $\mathcal{N} \mathcal{P}$ based on a particular exponential-time challenger assumption $(C, t)$, then the assumption can already be broken in polynomial time.

Moving on to non-interactive non-malleable commitments, we show that if non-malleability of a non-interactive, or two message, commitment scheme $\Pi$ can be based on a efficient-challenger (resp. $T(\cdot)$-size) challenger assumption $(C, t)$ using a polynomial-time (resp. $T(\cdot)$-sized) security reduction, then $C$ can be broken in polynomial-time (resp. by a poly $(T(\cdot))$-sized circuit).

Theorem 2 (Main Theorem 2-Informally stated). Let $\Pi$ be a two-message commitment scheme. Assume there exists a polynomial-time (resp. T(.)-size) Turing reduction $R$ such that $R^{A}$ breaks the efficient-challenger (resp. T(.)-size) assumption $C$ w.r.t. the threshold $t$ for every $A$ that breaks non-malleability of $\Pi$. Then $C$ can be broken in polynomial-time (resp. by a poly $(T(\cdot))$-sized circuit) with respect to the threshold $t$.

We emphasize that for all the above-mentioned results, the construction of the protocols $\Pi$ need not make use of the underlying assumption in a black-box way; the only restriction we impose is that the security reduction is a Turing (i.e., black-box) reduction.

Let us also remark that although we see only superficial similaries between the primitives of non-interactive statistical NIZK and non-interactive non-malleable commitments (e.g., they both refer to non-interactive primitives), the techniques used to prove the above impossibility results have significant overlap.

Uniform v.s. Non-uniform Security Reductions. In this work we focus on ruling out uniform security reductions; that is, the security reduction is a Turing machine that gets no advice about the attacker. Nevertheless, a very recent work by Chung, Lin, Mahmoody and Pass CLMP13 provides techniques for extending certain types of separation results for the uniform setting also to the non-uniform setting (where we consider reductions that may receive a polynomial-length advice about the attacker). These technique readily apply to our results, which thus also extend to rule out non-uniform security reductions. 
A Taxonomy of Intractability Assumption. As an independent contribution, we slightly generalize the notion of an intractability assumption from Pas11. (see also Nao03, DOP05, HH09, RV10, GW11) and provide an, in our eyes, natural taxonomy of intractability assumptions based on 1) the security threshold, 2) the number of communication rounds in the security game, 3) the computational complexity of the game challenger, 4) the communication complexity of the challenger, and 5) the computational complexity of the security reduction. Our results, combined with [Pas11, GW11, demonstrate several natural primitives that may be (trivially) based on assumption of a certain type (e.g., the soundness condition of a perfect NIZK can trivially be viewed as a bounded-round assumption), but cannot be based on a different type of assumption (e.g., an assumption where the challenger is efficient). Our results focus on understanding limitations in terms of items 1, 2, 3 and 5; we leave open an exploration of item 4, i.e., the communication complexity of the challenger. More generally, we are optimistic that cryptographic tasks may be classified in this taxonomy, based on whether they can be acheivedeven using a non-black-box construction - based on a class of assumptions in this taxonomy, but not on another (much like the celebrated taxonomy of Impagliazzo Imp95 in the context of black-box constructions.)

A Note on Random Oracles. Let us point out that in the Random Oracle model BR93, both of the above-mentioned primitives are easy to construct. Perfect NIZK were construced in BR93 (by relying on the "Fiat-Shamir heuristic" FS87) and non-interactive non-malleable commitments in Pas03a. Indeed, many practical protocols rely on the assumption that a "good" hashfunction behaves like a non-interactive non-malleable commitment, and on non-interactive zero-knowledge arguments constructed by applying the "Fiat-Shamir heuristic" [FS87 to a three-message perfect zero-knowledge protocol. Our results show that such commonly used sub-protocols cannot be proven secure based on standard hardness assumptions. Note that these results are incomparable to those of e.g., CGH04, GK03 on the "uninstantiability of random oracles": the results of CGH04, GK03 are stronger in the sense that any instantiation of their scheme with a concrete function can actually be broken, whereas we just show that the instantiated scheme cannot be proven secure using a Turing reduction based on standard assumptions. On the other hand, the separations of CGH04, GK03 consider "artifical protocols", whereas the protocols we consider are natural (and commonly used in practice).

\subsection{Related Separation Results}

There is a large literature on separation results between cryptographic primitives/assumptions. We distinguish between two types of results.

Separations for Fully Black-box Constructions. The seminal work of Impagliazzo and Rudich [R88] provides a framework for proving black-box separations between cryptographic primitives. We highlight that this framework considers so-called "fully-black-box constructions" (see RTV04 for a taxonomy of various black-box separations); that is, the framework considers both black-box 
constructions (i.e., the higher-level primitive only uses the underlying primitive as a black-box), and black-box reductions.

Separations for Black-box Reductions. In recent years, new types of black-box separations have emerged. These types of separation apply even to non-black-box constructions, but still only rule out black-box proofs of security: Pass Pas06. and Pass, Tseng and Venkitasubramaniam PTV11 (relying on the works of Brassard Bra83 and Akavia et al AGGM06, demonstrating limitations of "NP-hard Cryptography"2) demonstrate that under certain (new) complexity theoretic assumptions, various cryptographic task cannot be based on one-way functions using a black-box security reduction, even if the protocol uses the one-way function in a non-black-box way. Very recently, two independent works demonstrate similar types of separation bounds, but this time ruling our security reductions to a general set of intractability assumptions: Pass Pas11. demonstrates impossibility of using black-box reductions to prove the security of several primitives (e.g., Schnorr's identification scheme, commitment scheme secure under weak notions of selective opening, Chaum Blind signatures, etc) based on any "bounded-round" intractability assumption (where the challenger uses an a-priori bounded number of rounds, but is otherwise unbounded). Gentry and Wichs [GW11] demonstrate (assuming the existence of strong pseudorandom generators) impossibility of using black-box security reductions to prove soundness of "succinct non-interactive arguments" based on any "falsifiable" assumption (where the challenger is computationally bounded). Both of the above-mentioned work fall into the "meta-reduction" paradigm of Boneh and Venkatesan BV98, which was earlier used to prove separations for restricted types of reductions (see e.g., BMV08, HRS09, FS10]). Our separation results are in the vein of these two works, and follows some of their techniques.

\subsection{Proof Overview: Perfect NIZK with Adaptive Inputs}

Assume there exists a perfect $\operatorname{NIZK}(P, V)$ for a hard-on-the average language $L$; for simplicity, in this proof overview we focus on the case when the reference string is uniformly random (i.e., we consider only NIZK in the so-called Uniform Reference String (URS) Model). Assume further that there exists a Turing reduction $R$ such that $R^{A}$ breaks the assumption $C$ (with respect to some thresholds $t$ ) whenever $A$ breaks adaptive soundness of $(P, V)$. Following the "meta-reduction" paradigm by Boneh and Venkatesan BV98] (which is used in both Pas11] and GW11, and also AF07]), we want to use $R$ to directly break $C$.

More precisely (just as in Pas11, GW11) we exhibit a particular attacker $A$ to the adaptive soundness of $(P, V)$ and next show how to "emulate" this attacker for $R$ without disturbing $R$ 's interaction with $C$. Whereas in Pas11]

\footnotetext{
${ }^{2}$ See also the results of Feigenbaum and Fortnow [FF93] and the result of Bogdanov and Trevisan [BT03] that demonstrate limitations of NP-hard cryptography for restricted types of reductions.
} 
the emulation was statistically close (and thus the separation could be applied also to unbounded challengers), in GW11 the emulation was only computationally indistinguishable, but this still suffices for convincing $C$ as long as $C$ is computationally efficient. We here follow the approach of GW11.

Let us turn to describing our attacker $A$, and next explain how to emulate it. Given a $\operatorname{CRS} \rho, A$ first attempts to recover the random coins $r$ used by the simulator $S$ when outputting the $\operatorname{CRS} \rho$; since the simulation is perfect, such a string $r$ exists (but finding $r$ might require super-polynomial time). (Recall that since we are dealing with adaptive zero-knowledge, the zero-knowledge simulator needs to output a reference string $\rho$ before knowing what statement it needs to simulate a proof of.) Next, $A$ samples a false instance $x \notin L$ which is indistinguishable from a true instance (since $L$ is hard-on-the average, this can be done efficiently). Finally, it runs the simulator $S$ on the random coins $r$ to generate $\rho$, and next feeds it the instance $x$, and lets $\pi$ denote the proof output by $S$ (again this final step is efficient).

Let us argue that the proof $\pi$ of $x$ is accepted by $V(\rho)$. Towards this, consider a hybrid attacker $A^{\prime}$ that performs exactly the same steps as $A$, but instead samples a true instance $x \in L$. It follows from the ZK property (combined with the completeness property) that $V$ accepts the proofs output by $A^{\prime}$. Now, intuitively, it should follow from the hard-on-the-average property of $L$ that $V$ also accepts the proofs output by $A$. But there is a catch: recall that $A$ is not efficient. However, since it is only the first step of $A$ that is inefficient, we can fix the random string $r$ non-uniformly and still use the remaining steps of $A$ and the efficient verifier $V$ to contradict the hard-on-average property of $L$, as long as we assume that $L$ is hard-on-average for non-uniform polynomial-time. Note that we here rely on the fact that $A$ is allowed to choose the statement $x$ after having seen the reference string $\rho$ (i.e., we rely on $A$ breaking adaptive soundness) - this is what allows us to non-uniformly choose $r$ as a function of $\rho$, before sampling $x \in L$.

Now given this breaker $A$, let us see an attacker $\tilde{A}$ that efficiently simulates it (in a computationally indistinguishable way). $\tilde{A}(\rho)$ simply picks a random true statement $x$ together with a witness $w$, and next runs the honest prover strategy $P(\rho, x, w)$ to produce a proof $\pi$ (this strategy is similar to the one used in GW11]). It follows by the ZK property that the output of $C$ when communicating with $\tilde{A}$ and $A^{\prime}$ are indistinguishable, and we can then apply a similar argument as above (but more complicated) to argue that the output of $C$ when communicating with $A^{\prime}$ and $A$ are indistinguishable, and thus $R^{\tilde{A}}$ breaks $C$ with roughly the same probability as $R^{A}$ does.

Dealing with Exponential-time Challenger Assumptions. In case the runningtime of the challenger $C$ is super-polynomial in the security parameter $k$, the above approach seemingly fails: the fact that $\tilde{A}$ generates computationally indistinguishable messages does not suffice to argue that $C$ still accepts in the interaction with $R^{\tilde{A}}$. However, if we assume that the language $L$ is hard-on-the-average for non-uniform subexponential time, then the above approach still works, as long as $C$ is subexponential time; in fact, it rules out also subexponential-time 
reductions. To deal with also exponential-time challenger assumptions, we proceed as follows. If the same assumption $C$ can be used to prove any statement in $\mathcal{N P}$ of length polynomial in the security parameter, then if the language $L$ is hard-on-the-average for non-uniform sub-exponential time, it suffices to pick statements $x$ that are sufficiently long (but still of polynomial length) to ensure that $\tilde{A}$ generates messages that are indistinguishable from those sent by $\tilde{A}$, even by $C$.

\subsection{Proof Overview: Non-interactive Non-malleable Commitments}

Assume there exists a non-interactive commitment scheme $\Pi$; for simplicity of exposition we here focus only on non-interactive, as opposed to two-message, commitments. Assume further that there exists a Turing reduction $R$ such that $R^{A}$ breaks the assumption $C$ (with respect to some thresholds $t$ ) whenever $A$ breaks non-malleability of $\Pi$. Recall that an attacker $A$ that breaks nonmalleability of $\Pi$ participates in two interactions - one on the "left" acting as a receiver, and one on the "right" acting as a committer. To be successful $A$ needs to choose a different identity for the left and right interactions, and must commit to a value $\tilde{v}$ which is related to the value $v$ it receives a commitment to on the left. Consider a strong attacker $A$ that chooses identity 0 on the left, and 1 on the right, and upon receiving a commitment $c$ recovers (using brute force) the unique value $v$ that $c$ is a commitment to (if the value is not unique $v$ is set to $\perp$ ), and next honestly commits to $v$ on the right. Clearly $A$ breaks non-malleability of $\Pi$, and thus $R^{A}$ also breaks $C$ w.r.t. $t$.

Let us now see how to efficiently emulate $A$. We simply consider a "trivial" adversary $\tilde{A}$ that picks identity 0 on the left and 1 on the right (just as $A$ ), but instead of trying to commit to $v$ on the right, it simply commits to 0 on the right. Now, intuitively, if the reduction $R$ and the challenger $C$ are polynomial-time, then it should follow by the hiding property of $\Pi$ that $R^{\tilde{A}}$ still breaks $C$ (w.r.t. $t$ ). Note, however, that $R$ may be asking its oracle to break non-malleability of multiple commitments, and since $A$ is not efficiently computable, we need to be a bit careful when doing the hybrid argument. Nevertheless, using a careful ordering of the hybrid (and as in the lower bound for statistical NIZK) relying on the non-uniform hiding property of $\Pi$ we can show that $R^{\tilde{A}}$ still breaks $C$ (w.r.t. $t$ ).

Note that the above proof idea applies to a very weak notion of "one-sided" non-malleability, where the attacker always uses identity 0 on the left and 1 on the right; Liskov et al $\mathrm{LLM}^{+} 01$ call commitments satisfying this weak notion of non-malleability, mutually independent. Interestingly, [LLM+01] shows a construction of a mutually independent commitment based on the existence of subexponentially hard one-way permutations. The idea is simple: Let $\mathrm{Com}_{0}$ be a commitment scheme that is hard for subexponential time, and let $C o m_{1}$ be a commitment scheme that can be fully broken in subexponential time. If a MIM upon receving a commitment of $v$ using $\mathrm{Com}_{0}$ is able to output a commitment to a related value $\tilde{v}$ using $C o m_{1}$, then we can violate the hiding of $C o m_{0}$ by 
breaking $C \mathrm{Cm}_{1}$ using brute-force. This security reduction, however, is superpolynomial (subexponential) time. A natural question is thus whether subexponential time/size reductions may be helpful for constructing "full-fledged" (as opposed to one-sided) non-interactive commitments 3 We proceed to rule out such reductions (or rather to show that if there exists such a reduction, then the reduction itself must already break the assumption).

Consider a $T(k)$-sized reduction $R$, where $T(k)$ is super-polynomial, for basing non-malleability on an efficient challenger assumption $C_{4}$, and consider the algorithms $A$ and $\tilde{A}$ described above. Note that if $R$ has super-polynomial size, we have no guarantees that $R^{\tilde{A}}$ breaks $C$ even if $R^{A}$ does; since hiding of $\Pi$ is only required to hold for polynomial-sized algorithms, $R^{\tilde{A}}$ 's success probability may be very different from $R^{A}$ success probability. But in this case, intuitively, $R$ itself must be able to break the hiding of commitments using identity 1 (recall that $A$ and $\tilde{A}$ use identity 1 on the right).

So, if $R^{\tilde{A}}$ does not already convince $C$, we can use $R$ (in conjunction with $C$ ) to obtain a circuit $D$ that distinguishes, say commitments to $0^{k}$ and $1^{k}$ using identity 15 We may then use $D$ to construct a man-in-the-middle attacker $A^{\prime}$ that chooses identity 1 on the left and 0 on the right (as opposed to 0 on the left and 1 on the right, as $A$ and $\tilde{A}$ did) to break non-malleability of $\Pi$, and finally use $R$ combined with $A^{\prime}$ to directly break $C$. So, summarizing, either $R^{\tilde{A}}$ works, or else, we use $R$ in order to construct an MIM $A^{\prime}$ that breaks nonmalleability, and then use $R^{A^{\prime}}$ to convinve $C$-in essence, we use $R$ "on itself" to convince $C$.

\subsection{Overview of the Paper}

We provide definitions of intractability assumptions and black-box reductions in Section 2, this section also contains our taxonomy of intractability assumptions. We formally state and prove our results about NIZK in Section 3, A formal treatment of our results about non-malleable commitments are found in the full version.

\section{Intractability Assumptions and Black-box Reductions}

Our definition of an intractability assumption closely follows Pas11. Following Naor Nao03. (see also DOP05, HH09, RV10]), we model an intractability assumption as an interaction (or game) between a probabilistic machine $C$-called

\footnotetext{
${ }^{3}$ Indeed, [PW10] rely on intuitions similar to those from mutually independent commitments to construct a "full-fledged" non-malleable commitment, but this construction requires multiple communication rounds.

4 The assumption that $C$ is an efficient challenger is only made here to simplify exposition; our actual proof also works when $C$ is $T(k)$-sized.

${ }^{5}$ As in the previous proof, to obtain a machine that breaks the hiding of the commitment, we need to rely a polynomial-length non-uniform advice to deal with the above-mentioned inefficiency issue in the hybrid argument; this is why we work with circuits here.
} 
the challenger - and an attacker $A$. Both parties get as input $1^{k}$ where $k$ is the security parameter. Any such challenger $C$, together with a threshold function $t(\cdot)$ intuitively corresponds to the assumption:

For every polynomial-time adversary $A$, there exists a negligible function $\mu$ such that for all $k \in N$, the probability that $C$ outputs 1 after interacting with $A$ is bounded by $t(k)+\mu(k)$.

We say that $A$ breaks $C$ w.r.t $t$ with probability $p$ on common input $1^{k}$ if $\operatorname{Pr}\left[\langle A, C\rangle\left(1^{k}\right)=1\right] \geq t(k)+p$.

If the challenger $C$ is polynomial-time in the length of the messages it receives, we say that the assumption is efficient challenger; such assumptions are referred to as falsifiable assumptions by Naor [Nao03 and Gentry and Wichs [GW11. More generally, we refer to an assumption as having a $T(\cdot, \cdot)$-time (resp. size) challenger if $C$ can be implemented in time (resp. size) $T(k, \ell)$ on input the security parameter $1^{k}$, and when receiving messages of length $\ell$. $(C, t)$ is an efficient challenger assumption iff $C$ is a $T(\cdot, \cdot)$-assumption where $T(k, \ell)$ is polynomial in both $k$ and $\ell$. For simplicity, we here consider either poly $(k, \ell)$-time (or size) challengers, or $T(k, \ell)=T(k)$-time (or size) challengers, where the running-time of the challenger is bounded only as a function of the security parameter.

We can easily model all "traditional" cryptographic assumptions as efficient challengers $C$ and a threshold $t$. For instance, the assumption that a particular function $f$ is (strongly) one-way corresponds to the threshold $t(k)=0$ and the 2round challenger $C$ that on input $1^{k}$ pick a random input $x$ of length $k$, sends $f(x)$ to the attacker, and finally outputs 1 iff the attacker returns an inverse to $f(x)$. Decisional assumptions (such as, e.g., the decisional Diffie-Hellman problem, or the assumption that a particular function $g$ is a pseudorandom generator) can also easily be modelled as 2-round challengers but now we have the threshold $t(k)=1 / 2$. More esoteric assumptions such as the "one-more discrete logarithm assumption" BNPS03, BP02, or "adaptive one-way functions" [PPV08, are not efficient-challenger assumptions; however, they can be modeled as exponentialtime challenger assumptions.

We may also consider other restricted types of intractability assumptions. For instance, Pas11 considers challengers $C$ that are computationally unbounded, but for which there exists a polynomial upper bound in the terms of the security parameter $k$ on the number of communications rounds by $C$; we refer to these assumptions as bounded round intractability assumptions. Another interesting class of assumptions is obtained by further restricting the communication complexity of $C$; for instance, we may require that there is a polynomial bound (again in terms of the security parameter $k$ ) on the communication complexity of $C$; we refer to these assumptions as bounded-communication intractability assumption.

In this work we focus on establishing impossibility results for reductions from efficient-challenger (just as the results of GW11]), and more generally time/size $T(\cdot)$ challenger assumptions where $T(\cdot)$ is a super-polynomial function. As mentioned, in Pas11] impossibility results for reductions from bounded-round assumptions are presented. Since both non-malleability of a protocol, and 
adaptive soundness of a NIZK, is a bounded round assumption, we cannot hope to strengthen our result to rule out reductions also from bounded round assumptions. We leave open an exploration of bounded-communication intractability assumptions.

Finally, note that we can capture super-polynomial hardness of an assumption by allowing for super-polynomial-time reductions to the assumption.

A Taxonomy of Intractability Assumption. The above way of modeling assumptions, provides an, in our eyes, natural taxonomy of intractability assumptions based on 1) the security threshold $t, 2)$ the number of communication rounds used by $C, 3)$ the computational complexity of $C, 4)$ the communication complexity of $C$, and 5) the computational complexity of the security reduction. We are optimistic that cryptographic tasks may classified in this taxonomy, based on whether they can be acheived-even using a non-black-box constructionbased on class of assumptions in this taxonomy, but not on another (much like the celebrated taxonomy of Impagliazzo Imp95] in the context of black-box constructions.)

Indeed, as mentioned above, the results of Pas11, GW11] already yield some results in this direction, separating unbounded-round and bounded-round assumptions Pas11 and unbounded-challenger and efficient-challenger assumptions GW11. The results in this paper further elucidate the landscape; among other things, separating unbounded challenger and exponential-time challenger assumptions, and exponential-time and efficient-challenger assumptions.

An interesting question for future work is obtaining separations for nonblack-box constructions for more "structured" types of assumptions (such as the existence of one-way functions, one-way permutations). The results of Pas06. PTV11 provide a first step in this direction, exhibiting separations from one-way functions for some natural cryptographic primitives, but rely on new complexitytheoretic assumptions.

Black-box Reductions. We consider probabilistic polynomial time Turing reductions - i.e., black-box reductions. A black-box reduction refers to a probabilistic polynomial-time oracle algorithm. Roughly speaking, a black-box reduction for basing the security of a primitive $P$ on the hardness of an assumption $C$, is a probabilistic polynomial-time oracle machine $R$ such that whenever the oracle $O$ "breaks" $P$ with respect to the security parameter $k$, then $R^{O}$ "breaks" $C$ with respect to a polynomially related security parameter $k^{\prime}$ such that $k^{\prime}$ can be efficiently computed given $k$. We restrict to the case when $k^{\prime}=k$. This is without loss of generality: we can always redefine the assumption $C$ so that it on input $k$ acts as if its input actually was $k^{\prime}$ (since $k^{\prime}$ can be efficiently computed given $k$ ). To formalize this notion, we thus restrict to oracle machines $R$ that on input $1^{k}$ always query their oracle on inputs $\left(1^{k}, \cdot\right)$.

Definition 1. We say that $R$ is a valid black-box reduction if $R$ is an oracle machine such that $R\left(1^{k}\right)$ only queries its oracle with inputs of the form $\left(1^{k}, y\right)$, where $y \in\{0,1\}^{*}$. 
The reason that we (and as it standard in the literature) restrict $R$ to only query its oracle on a single "input length" $k$, is that standard cryptographic definitions require ruling out the existence of attackers that break some primitive even for infinitely many input lengths; as these inputs lengths can be very sparse, a blackbox reduction must be successful even if it has access to an attacker that only succeeds on a single input length 6

\section{Security of Perfect Adaptive NIZK}

We recall the traditional definition of non-interactive proofs in the Common Reference String (CRS) model. For generality (and since we are proving a lower bound) we allow the CRS $\rho$ be generated by an arbitrary polynomial-time distribution (as opposed to requiring it to be uniformly random). In the adaptivelysound notion of an non-interactive proof/argument, we require that soundness holds even if the attacker may adaptively pick a statement after having seen the CRS. We here focus only on proofs for languages in $\mathcal{N} \mathcal{P}$ where the prover is efficient when given an $\mathcal{N} \mathcal{P}$-witness.

Definition 2 (Non-Interactive Proofs/Arguments). A triple of algorithms, $(\mathcal{D}, P, V)$, is called a non-interactive proof system (with non-adaptive soundness) for a language $L$ if the algorithm $\mathcal{D}$ is probabilistic polynomial-time, the algorithm $V$ is a deterministic polynomial-time, and $P$ is probabilistic polynomialtime, such that the following two conditions hold:

- Completeness: There exists a negligible function $\mu$ such for every $x \in L$, every $w \in R_{L}(x)$ and every $k \in N$,

$$
\operatorname{Pr}\left[\rho \leftarrow \mathcal{D}\left(1^{k}, 1^{|x|}\right) ; \pi \leftarrow P\left(1^{k}, x, w, \rho\right): V\left(1^{k}, x, \rho, \pi\right)=1\right] \geq 1-\mu(k)
$$

- Soundness: For every algorithm $B$ and every polynomial $q$, there exists a negligible function $\mu$ such that for every $k \in N$ and every $x \notin L$ such that $|x| \leq q(k)$

$$
\operatorname{Pr}\left[\rho \leftarrow \mathcal{D}\left(1^{k}, 1^{|x|}\right) ; \pi^{\prime} \leftarrow B\left(1^{k}, x, \rho\right): V\left(1^{k}, x, \rho, \pi^{\prime}\right)=1\right] \leq \mu(k)
$$

If additionally the following condition holds, then we call $(\mathcal{D}, P, V)$ an adaptivelysound non-interactive proof system:

- Adaptive Soundness: For every algorithm $B$ and every polynomial $q$, there exists a negligible function $\mu$ such that for every $k \in N, n \in[q(k)]$

$$
\operatorname{Pr}\left[\begin{array}{l}
\left.\rho \leftarrow \mathcal{D}\left(1^{k}, 1^{n}\right) ;\left(x, \pi^{\prime}\right) \leftarrow B\left(1^{k}, 1^{n}, \rho\right): V\left(1^{k}, x, \rho, \pi^{\prime}\right)=1\right] \leq \mu(k) \\
\wedge|x|=n \wedge x \notin L
\end{array}\right.
$$

\footnotetext{
${ }^{6}$ For instance, consider an attacker that succeeds only on input lengths $2^{c}, 2^{2^{c}}, \ldots$ (and outputs $\perp$ on all other inputs); a black-box reduction that only accesses its oracle on a polynomially related security parameter can only access a single "non- $\perp$ " input length
} 
Finally, if the soundness (resp adaptive soundness) condition only holds w.r.t polynomial-time adversaries $B$, we call $(\mathcal{D}, P, V)$ a non-interactive argument (resp. an adaptively-sound non-interactive argument)).

Let us turn to defining zero-knowledge. Also here there is a non-adaptive and an adaptive version. In the non-adaptive definition of zero-knowledge from BFM88, there is a single simulator, which, after seeing the statement to be proven, generates both the CRS and the proof at the same time. In the adaptive definition from [FLS90, there are two simulators - the first of which must output a string before seeing any theorems. The stronger adaptive definition guarantees zero-knowledge even when the statement to be proved is chosen as a function of the CRS. We here focus only on adaptive zero-knowledge.

Definition 3 (Non-Interactive Zero-Knowledge). Let $(\mathcal{D}, P, V)$ be an noninteractive proof system for the language $L$. We say that $(\mathcal{D}, P, V)$ is (adaptively) zero-knowledge if there exists two probabilistic polynomial-time simulators $S_{1}, S_{2}$ such that for every polynomial $q$, every non-uniform polynomial-time statementchosing algorithm $c(\cdot)$ that on input $\left(1^{k}, 1^{n}, \rho\right)$ outputs a $n$-bit statement $x \in L$, and every function $w(\cdot)$ such that $w(x) \in R_{L}(x)$, the following two ensembles are computationally indistinguishable

$$
\begin{aligned}
& \left\{\rho \leftarrow \mathcal{D}\left(1^{k}, 1^{n}\right) ; x \leftarrow c\left(1^{k}, 1^{n}, \rho\right) ; w \leftarrow w(x) ; \pi \leftarrow P\left(1^{k}, x, w, \rho\right):(\rho, x, \pi)\right\}_{k \in N, n \in[q(k)]} \\
& \left\{(\rho, \operatorname{aux}) \leftarrow S_{1}\left(1^{k}, 1^{n}\right) ; x \leftarrow c\left(1^{k}, 1^{n}, \rho\right) ; \pi^{\prime} \leftarrow S_{2}\left(1^{k}, x, \text { aux }\right):\left(\rho, x, \pi^{\prime}\right)\right\}_{k \in N, n \in[q(k)]}
\end{aligned}
$$

We furthermore say that $(\mathcal{D}, P, V)$ is perfect (resp. statistical) zero-knowledge if the above ensembles are identically distributed (resp. statistically close).

We use the (common) acronym "NIZK" to denote a non-interactive zeroknowledge proof or argument. Feige, Lapidot and Shamir and Bellare and Yung FLS90, BY96, (building on BFM88]) show that the existence of enhanced trapdoor permutations implies that all of $\mathcal{N} \mathcal{P}$ has a adaptively-sound NIZK, but the zero-knowledge property is only computational. As mentioned, Groth, Ostrovsky and Sahai GOS06 show (under some number theoretic assumptions) that all of $\mathcal{N} \mathcal{P}$ has a perfect NIZK with non-adaptive soundness. More recently, Abe and Fehr [AF07] present a perfect NIZK for $\mathcal{N} \mathcal{P}$ also with adaptive soundness but based the soundness property on a "knowledge" assumption (rather than an intractability assumption).

We aim to prove limitations of basing even weak notions of adaptive soundness for perfect or statistical NIZK for $\mathcal{N} \mathcal{P}$ on intractability assumptions. Let us first explicitly define what it means to break adaptive soundnessof a NIZK.

Definition 4 (Breaking Adaptive Soundness). We say that $A$ breaks adaptive soundness of $(\mathcal{D}, P, V)$ w.r.t the language $L$ on input lengths $q(\cdot)$ with probability $\mu(\cdot)$ if for every $k \in N$,

$$
\operatorname{Pr}\left[\begin{array}{l}
\rho \leftarrow \mathcal{D}\left(1^{k}, 1^{q(k)}\right) ;\left(x, \pi^{\prime}\right) \leftarrow A\left(1^{k}, \rho\right): V\left(1^{k}, x, \rho, \pi^{\prime}\right) \wedge|x| \\
=q(k) \wedge x \notin L=1
\end{array}\right] \geq \mu(k)
$$

Let us turn to defining what it means to base adaptive soundness on an intractability assumption $C$. 
Definition 5 (Basing Adaptive Soundness on the Hardness of $C$ ). We say that $R$ is a black-box reduction for basing adaptive soundness of $(\mathcal{D}, P, V)$ w.r.t. $L$ and input lengths $q$, on the hardness of $C$ w.r.t threshold $t(\cdot)$ if $R$ is a valid blackbox reduction and there exists a polynomial $p(\cdot, \cdot)$ such that for every probabilistic machine $A$ that breaks adaptive soundness of $(\mathcal{D}, P, V)$ w.r.t $L$ and inputs lengths $q(\cdot)$ with probability $\mu(\cdot)$, for every $k \in N, R^{A}$ breaks $C$ w.r.t $t$ with probability $p(\mu(k), 1 / k)$ on input $1^{k}$.

Note that we here require that $R^{O}$ breaks the assumption $C$ on the security parameter $k$ by querying $O$ on the same security parameter $k$. As previously mentioned, a seemingly more general definition would allow $R^{O}$ to break $C$ on a polynomially-related security parameter $k^{\prime}$ (which can be efficiently computed given $k$ ), but this extra generality does not buy us anything as we can always re-define $C$ to on input $k$ act as its input was $k^{\prime}$.

We now have the following theorem:

Theorem 3. Assume the existence of non-uniformly hard one-way functions. Then there exists an $\mathcal{N} \mathcal{P}$-language $L$ such that the following holds. Let $(\mathcal{D}, P, V)$ be a statistical non-interactive adaptively zero-knowledge argument for $L$, let $q(k)$ be polynomially related to $k$, and let $(C, t)$ be any efficient-challenger assumption. If there exists a black-box reduction $R$ for basing adaptive soundness of $(\mathcal{D}, P, V)$ w.r.t $L$ and input lengths $q$ on the hardness of $C$ w.r.t threshold $t$, then there exists a probabilistic polynomial-time machine $B$ and a polynomial $p^{\prime}(\cdot)$ such that for infinitely many $k \in N, B$ breaks $C$ w.r.t $t$ with probability $\frac{1}{p^{\prime}(k)}$ on input $1^{k}$. If furthermore assuming the existence of one-way functions secure against non-uniform subexponential-time algorithms, the above holds even if $C$ is subexponential-time computable.

Let us also remark that under the assumption of one-way functions secure against non-uniform subexponential-time algorithms, Theorem 3 directly extends also to a super-polynomial-time (SPS) Pas03b relaxation of the notion of a statistical NIZK, where the simulator may run in subexponential time. (Let us also briefly point our a very recent work by Chung, Lui, Mohammad and Pass CLMP12] that presents barriers to two-message SPS zero-knowledge arguments.)

Note that in Theorem 3, we rule out statistical NIZK where adaptive soundness only needs to hold w.r.t. statements of a particular (polynomial) length $n=q(k)$.

Our next theorem rules out even exponential-time challenger assumptions $C$ if the same assumption $C$ can be used to prove adaptive soundness for any polynomial length statement (indeed, as far as we know, in all known NIZK constructions, the underlying intractability assumption depends only on the security parameter for the NIZK but not on the length of the statement to be proven).

Theorem 4. Assume the existence of one-way functions secure against nonuniform subexponential-time algorithms. Then there exists an $\mathcal{N P}$-language $L$ such that the following holds. Let $(\mathcal{D}, P, V)$ be a statistical non-interactive adaptively zero-knowledge argument for $L$, and let $(C, t)$ be any exponential-time challenger assumption. If for every polynomial $q$, there exists a black-box reduction $R$ 
for basing adaptive soundness of $(\mathcal{D}, P, V)$ w.r.t $L$ and the input length $q$ on the hardness of $C$ w.r.t threshold $t$, then there exists a probabilistic polynomial-time machine $B$ and a polynomial $p^{\prime}(\cdot)$ such that for infinitely many $k \in N, B$ breaks $C$ w.r.t $t$ with probability $\frac{1}{p^{\prime}(k)}$ on input $1^{k}$.

Note that Theorem 4 is weaker than Theorem 3 in that we require that the same assumption $C$ can be used to prove any polynomial-length statement, whereas in Theorem 3 we rule out NIZK where the underlying hardness assumption may depend also on the length of the statement proved. This additional restriction is necessary: the assumption that a particular NIZK is adaptively sound for statements of length $q(k)=k$ can clearly be stated as an exponential-time challenger assumption.

We here only prove Theorem 3 and leave Theorem 4 for the full version.

\subsection{Proof of Theorem 3}

Proof. We here consider only a simplified case when the zero-knowledge property is perfect and the distribution sampled by $\mathcal{D}$ is uniform over $\{0,1\}^{\text {poly }(k) \text {-i.e., }}$ we consider perfect NIZK in the so-called "Uniform Reference String" (URS) model. The remainder of the proof of Theorem can be found in the full version. Let $g:\{0,1\}^{*} \rightarrow\{0,1\}^{*}$ be a length-doubling PRG. Consider the language $L=$ $\left\{g(s) \mid s \in\{0,1\}^{*}\right\}$. Assume there exists a perfect $\operatorname{NIZK}(\mathcal{D}, P, V)$ for $L$ in the URS model, where the reference string is of length $\ell(k)$ given the security parameter $k$, and assume there exists a black-box reduction $R$ for basing adaptive soundness of $(\mathcal{D}, P, V)$ w.r.t $L$ and input lengths $q(\cdot)$ on the hardness of $C$ w.r.t threshold $t$. In particular, this means that for every $A$ that breaks the adaptive soundness of $(\mathcal{D}, P, V)$ w.r.t $L$ and input lengths $q(\cdot)$ with overwhelming probability, there exists a polynomial $p(\cdot)$ such that for infinitely many $k \in N, R^{A}$ breaks $C$ w.r.t $t$ on common input $1^{k}$ with probability $\frac{1}{p(k)}$; i.e., $\operatorname{Pr}\left[\left\langle R^{A}, C\right\rangle\left(1^{k}\right)=1\right] \geq$ $t(k)+\frac{1}{p(k)}$

To be more concrete, $R$ may feed $A\left(1^{k}\right)$ a reference string $\rho$, and will get in return a statement $x \in\{0,1\}^{q(k)}$ — that with high probability is false-and a proof $\pi$ of $x$-that with high probability is accepting; $R$ may continue this process all throughout its interaction with $C$. Note that $R$ is required to work even if $A$ is probabilistic, and on each query made by $R, A$ uses fresh random coins. (As we show in the full version, at the cost of a minor complication, the proof can be adapted to work also if only considering reductions that work as long as the attacker is deterministic.)

Our goal is to present a polynomial-time algorithm that directly breaks $C$ without the help of $A$. Towards this goal, we will first define a particular randomized attacker $A$, and next present an efficient "simulator" $\tilde{A}$ for $A$, and show that $R^{\tilde{A}}$ still breaks $C$ (w.r.t $t$ ).

Let us start by defining the attacker $A$. To simplify notation, let us assume that $q(k)=2 k$; it is easy to see that the same proof works as long as $q(k)$ is polynomially related to $k$. On input $1^{k}$ and a reference string $\rho, A$ proceeds as follows: 
- $A$ first checks that $|\rho|=\ell(k)$; if not, it simply sends back $\perp$.

- Otherwise, it uniformly picks a random tape $r$ such that $S_{1}\left(1^{k}, 1^{n}\right)$ outputs $\rho$, aux on input the random tape $r$. Since, by our assumption, the simulation is perfect, every string $\rho \in\{0,1\}^{\ell(k)}$ is output by $S_{1}\left(1^{k}, 1^{n}\right)$ with positive (and the same) probability, so $A$ will succeed in this task. Note, however, that this step is not necessarily efficient.

- Next, $A$ uniformly picks a string $x \in\{0,1\}^{n}$. Note that, except with probability $2^{-k}, x \notin L$ (there are $2^{2 k}$ strings, and at most $2^{k}$ can be in the range of the PRG $g$ ).

- Finally, $A$ runs the simulator $S_{2}\left(1^{k}, 1^{n}, x\right.$, aux $)$ to produce the proof $\pi$, and outputs $(x, \pi)$.

As noted above, with high probability, the statement $x$ picked by $A$ is false. But it remains to argue that the proof $\pi$ of $x$ output by $A$ is accepting (for the reference string $\rho$ ). For now, let us simply assume that the proof is accepting with high probability, and let instead show how to emulate $A$ in polynomial time (thus proving that $C$ can be broken in polynomial time). We will then return to showing that $A$ indeed is a "good" attacker, producing accepting proofs of false statements.

Consider the "simulator", $\tilde{A}$ that on input $1^{k}$ and a reference string $\rho$, proceeds as follows:

- Just as $A, \tilde{A}$ first check that $|\rho|=\ell(k)$; if not it simply sends back $\perp$.

- Next, $\tilde{A}$ uniformly picks a string $s \in\{0,1\}^{k}$, and lets $x=g(s)$. Note that by definition $x \in L$.

- Finally, $\tilde{A}$ runs the honest prover algorithm $P\left(1^{k}, \rho, x, w\right)$ to produce the proof $\pi$, and outputs $(x, \pi)$.

The following claim shows that $\tilde{A}$ is a good simulator for $A$.

Claim 1. For every efficient $C$ and $R$, there exists a negligible function $\mu$ such that for every $k \in N,\left|\operatorname{Pr}\left[\left\langle R^{\tilde{A}}, C\right\rangle\left(1^{k}\right)=1\right]-\operatorname{Pr}\left[\left\langle R^{A}, C\right\rangle\left(1^{k}\right)=1\right]\right| \leq \mu(k)$.

Proof. As a first attempt to proving the claim, consider a hybrid attacker $A^{\prime}$ that performs exactly the same steps as $A$, but samples a true statement $x \in L$ in exactly the same way as $\tilde{A}$ (but otherwise runs the simulator, just as $A$ ). Note that the only difference between $A^{\prime}$ and $\tilde{A}$ is that $A^{\prime}$ provides "simulated" proofs (of true statements), whereas $\tilde{A}$ gives honestly generated proofs. Indeed, it follows from the perfect zero-knowledge property that $A^{\prime}$ perfectly emulates $\tilde{A}$. Furthermore, intuitively, it should follows from the fact that true and false statement are indistinguishable (by the pseudorandomness property of $g$ ) that $A^{\prime}$ correctly emulates $A$. But there is a problem: although, both $C$ and $R$ are efficient, $A$ and $A^{\prime}$ are not, so efficiently contradicting the pseudorandomness property becomes problematic.

To circumvent this problem, we define a carefully ordered sequence of hybrid experiments, and rely on the fact that it is only the first step of $A$ (and $A^{\prime}$ ) that is inefficient. With this careful ordering, the inefficient part of $A$ can be dealt 
with using non-uniformity (and thus we finally contradict the pseudorandomness property of $g$ w.r.t. non-uniform polynomial-time algorithms).

More precisely, assume for contradiction that the claim is false. That is, there exists a polynomial $p^{\prime}$ such that for infinitely many $k \in N$, $\left|\operatorname{Pr}\left[\left\langle R^{\tilde{A}}, C\right\rangle\left(1^{k}\right)=1\right]-\operatorname{Pr}\left[\left\langle R^{A}, C\right\rangle\left(1^{k}\right)=1\right]\right| \geq \frac{1}{p^{\prime}(k)}$. Let $m(k)$ be an upperbound on the number of oracle queries by $R$ on input $1^{k}$, and fix a canonical $k$ for which the above happens. Consider a sequence of hybrid experiments $H_{0}, \ldots, H_{m(k)}$, where $H_{i}$ is defined as the output of $C\left(1^{k}\right)$ after communicating with $R\left(1^{k}\right)$ where the first $i$ oracle queries of $R$ are answered by $A$, and the remaining ones are answered by the efficient $\tilde{A}$. Note that $H_{0}=\left\langle R^{\tilde{A}}, C\right\rangle\left(1^{k}\right)$ and $H_{m(k)}=\left\langle R^{A}, C\right\rangle\left(1^{k}\right)$. It follows that there exists some $j$ such that $\left|\operatorname{Pr}\left[H_{j+1}=1\right]-\operatorname{Pr}\left[H_{j}=1\right]\right| \geq \frac{1}{m(k) p^{\prime}(k)}$. Define another hybrid $H_{j}^{\prime}$ which is identically defined to $H_{j}$, but where the statement $x$ in the $j+1$ oracle query is selected as a true statement (just as in $H_{j+1}$ ) but we still run the simulation (just as in $\left.H_{j}\right)$. It follows directly by the perfect zero-knowledge property of $(\mathcal{D}, P, V)$ that the output of $H_{j}^{\prime}$ is identically distributed to the output of $H_{j+1}$. To reach a contradiction, let us finally argue that the output of $H_{j}$ is indistinguishable to that of $H_{j}^{\prime}$. Note that up until the point when $R$ receives its $(j+1)$ st proof back from the oracle, the two experiments proceed identically the same. Thus, if they are distinguishable, there exists some prefix $\tau$ of the execution of $H_{j} 7$, up until and including the $j+1$ query of $R$, such that conditioned on this prefix $\tau, H_{j}$ and $H_{j}^{\prime}$ are distinguishable. We may now simply extend $\tau$ to also include the string aux picked by $A$ in the $j+1$ query, and conclude that there exists some extension $\tau^{\prime}$ of $\tau$ such that even conditioned on $\tau^{\prime}, H_{j}$ and $H_{j}^{\prime}$ are distinguishable. But now, note that given the prefix $\tau^{\prime}$, the continuations of $H_{j}$ and $H_{j}^{\prime}$ (conditioned on $\tau^{\prime}$ ) can be efficiently generated. And since the only difference between them is the choice of the statement $x$, if they can be distinguished, we violate the pseudorandomness property of $g$. Note that we here require that $g$ is pseudorandom against non-uniform polynomial time (as we need the non-uniform advice $\tau^{\prime}$ ). This concludes the proof of Claim 1 .

So conclude the proof of the theorem, it only remains to show that $A$ is a good attacker. Note that by the completeness property of $(\mathcal{D}, P, V)$ it holds that, except with negligible probability, $\tilde{A}$ provides accepting proofs. It now follows as a corollary of Claim 1 that except with negligible probability, $A$ also provides accepting proofs: simply let $R$ be the reduction that picks an honestly generated reference string $\rho$, and upon receiving back the pair $(x, \pi)$, outputs 1 iff $V\left(1^{k}, \rho, x, \pi\right)$ outputs 1 , and let $C$ be the algorithm that simply outputs whatever $R$ outputs.

Ruling out Subexponential-time Challenger Assumptions. If the challenger $C$ is not efficient, then in the above hybrid argument, when switching the statement $x=g(s)$ from being pseudorandom to being truly random, we can no longer

${ }^{7}$ Technically, the prefix includes the random tape of $C$ and $R$ and all the answers to the first $j$ queries by $R$. 
directly argue that the probability of $C$ outputting 1 does not change by much. However, if use a PRG secure against subexponential time, then same proof goes through as long as $C$ is subexponential-time computable.

Acknowledgements. I am extremely grateful to Kai-min Chung and Mohammad Mahmoody for many helpful comments and definitional discussions.

\section{References}

[AF07] Abe, M., Fehr, S.: Perfect NIZK with Adaptive Soundness. In: Vadhan, S.P. (ed.) TCC 2007. LNCS, vol. 4392, pp. 118-136. Springer, Heidelberg (2007)

[AGGM06] Akavia, A., Goldreich, O., Goldwasser, S., Moshkovitz, D.: On basing oneway functions on NP-hardness. In: STOC 2006, pp. 701-710 (2006)

[Bar02] Barak, B.: Constant-round coin-tossing with a man in the middle or realizing the shared random string model. In: FOCS 2002: Proceedings of the 43rd Symposium on Foundations of Computer Science, pp. 345-355. IEEE Computer Society, Washington, DC (2002)

[BFM88] Blum, M., Feldman, P., Micali, S.: Non-interactive zero-knowledge and its applications (extended abstract). In: STOC, pp. 103-112 (1988)

[BMV08] Bresson, E., Monnerat, J., Vergnaud, D.: Separation Results on the "OneMore" Computational Problems. In: Malkin, T. (ed.) CT-RSA 2008. LNCS, vol. 4964, pp. 71-87. Springer, Heidelberg (2008)

[BNPS03] Bellare, M., Namprempre, C., Pointcheval, D., Semanko, M.: The onemore-rsa-inversion problems and the security of chaum's blind signature scheme. J. Cryptology 16(3), 185-215 (2003)

[BP02] Bellare, M., Palacio, A.: GQ and Schnorr Identification Schemes: Proofs of Security against Impersonation under Active and Concurrent Attacks. In: Yung, M. (ed.) CRYPTO 2002. LNCS, vol. 2442, pp. 162-177. Springer, Heidelberg (2002)

[BR93] Bellare, M., Rogaway, P.: Random oracles are practical: A paradigm for designing efficient protocols. In: ACM Conference on Computer and Communications Security, pp. 62-73 (1993)

[Bra83] Brassard, G.: Relativized cryptography. IEEE Transactions on Information Theory 29(6), 877-893 (1983)

[BT03] Bogdanov, A., Trevisan, L.: On worst-case to average-case reductions for np problems. In: FOCS, pp. 308-317 (2003)

[BV98] Boneh, D., Venkatesan, R.: Breaking RSA May Not Be Equivalent to Factoring. In: Nyberg, K. (ed.) EUROCRYPT 1998. LNCS, vol. 1403, pp. 59-71. Springer, Heidelberg (1998)

[BY96] Bellare, M., Yung, M.: Certifying permutations: Noninteractive zeroknowledge based on any trapdoor permutation. J. Cryptology 9(3), 149-166 (1996)

[CGH04] Canetti, R., Goldreich, O., Halevi, S.: The random oracle methodology, revisited. J. ACM 51(4), 557-594 (2004)

[CIO98] Di Crescenzo, G., Ishai, Y., Ostrovsky, R.: Non-interactive and nonmalleable commitment. In: STOC, pp. 141-150 (1998)

[CLMP12] Chung, K.-M., Lui, E., Mahmoody, M., Pass, R.: Unprovable security of two-message zero-knowledge (2012) 
[CLMP13] Chung, K.-M., Lin, H., Mahmoody, M., Pass, R.: On the power of nonuniform proof of security. In: ITCS 2013 (2013)

[Dam91] Damgård, I.B.: Towards Practical Public Key Systems Secure against Chosen Ciphertext Attacks. In: Feigenbaum, J. (ed.) CRYPTO 1991. LNCS, vol. 576, pp. 445-456. Springer, Heidelberg (1992)

[DDN00] Dolev, D., Dwork, C., Naor, M.: Nonmalleable cryptography. SIAM Journal on Computing 30(2), 391-437 (2000)

[DOP05] Dodis, Y., Oliveira, R., Pietrzak, K.: On the Generic Insecurity of the Full Domain Hash. In: Shoup, V. (ed.) CRYPTO 2005. LNCS, vol. 3621, pp. 449-466. Springer, Heidelberg (2005)

[FF93] Feigenbaum, J., Fortnow, L.: Random-self-reducibility of complete sets. SIAM Journal on Computing 22(5), 994-1005 (1993)

[FLS90] Feige, U., Lapidot, D., Shamir, A.: Multiple non-interactive zero knowledge proofs based on a single random string. In: FOCS 1990, pp. 308-317 (1990)

[FS87] Fiat, A., Shamir, A.: How to Prove Yourself: Practical Solutions to Identification and Signature Problems. In: Odlyzko, A.M. (ed.) CRYPTO 1986. LNCS, vol. 263, pp. 186-194. Springer, Heidelberg (1987)

[FS10] Fischlin, M., Schröder, D.: On the Impossibility of Three-Move Blind Signature Schemes. In: Gilbert, H. (ed.) EUROCRYPT 2010. LNCS, vol. 6110, pp. 197-215. Springer, Heidelberg (2010)

[GK03] Goldwasser, S., Kalai, Y.T.: On the (in)security of the fiat-shamir paradigm. In: FOCS 2003, pp. 102-111 (2003)

[GOS06] Groth, J., Ostrovsky, R., Sahai, A.: Perfect Non-interactive Zero Knowledge for NP. In: Vaudenay, S. (ed.) EUROCRYPT 2006. LNCS, vol. 4004, pp. 339-358. Springer, Heidelberg (2006)

[Goy11] Goyal, V.: Constant round non-malleable protocols using one way functions. In: STOC, pp. 695-704 (2011)

[GW11] Gentry, C., Wichs, D.: Separating succinct non-interactive arguments from all falsifiable assumptions. In: STOC, pp. 99-108 (2011)

[HH09] Haitner, I., Holenstein, T.: On the (Im)Possibility of Key Dependent Encryption. In: Reingold, O. (ed.) TCC 2009. LNCS, vol. 5444, pp. 202-219. Springer, Heidelberg (2009)

[HRS09] Haitner, I., Rosen, A., Shaltiel, R.: On the (Im)Possibility of ArthurMerlin Witness Hiding Protocols. In: Reingold, O. (ed.) TCC 2009. LNCS, vol. 5444, pp. 220-237. Springer, Heidelberg (2009)

[Imp95] Impagliazzo, R.: A personal view of average-case complexity. In: Structure in Complexity Theory 1995, pp. 134-147 (1995)

[IR88] Impagliazzo, R., Rudich, S.: Limits on the Provable Consequences of One-Way Permutations. In: Goldwasser, S. (ed.) CRYPTO 1988. LNCS, vol. 403, pp. 8-26. Springer, Heidelberg (1990)

$\left[\mathrm{LLM}^{+} 01\right]$ Liskov, M., Lysyanskaya, A., Micali, S., Reyzin, L., Smith, A.: Mutually Independent Commitments. In: Boyd, C. (ed.) ASIACRYPT 2001. LNCS, vol. 2248, pp. 385-401. Springer, Heidelberg (2001)

[LP09] Lin, H., Pass, R.: Non-malleability amplification. In: STOC 2009, pp. 189198 (2009)

[LP11] Lin, H., Pass, R.: Constant-round non-malleable commitments from any one-way function. In: STOC, pp. 705-714 (2011)

[LPV08] Lin, H., Pass, R., Venkitasubramaniam, M.: Concurrent Non-malleable Commitments from Any One-Way Function. In: Canetti, R. (ed.) TCC 2008. LNCS, vol. 4948, pp. 571-588. Springer, Heidelberg (2008) 
[Nao03] Naor, M.: On Cryptographic Assumptions and Challenges. In: Boneh, D. (ed.) CRYPTO 2003. LNCS, vol. 2729, pp. 96-109. Springer, Heidelberg (2003)

[Pas03a] Pass, R.: On Deniability in the Common Reference String and Random Oracle Model. In: Boneh, D. (ed.) CRYPTO 2003. LNCS, vol. 2729, pp. 316-337. Springer, Heidelberg (2003)

[Pas03b] Pass, R.: Simulation in Quasi-Polynomial Time, and its Application to Protocol Composition. In: Biham, E. (ed.) EUROCRYPT 2003. LNCS, vol. 2656, pp. 160-176. Springer, Heidelberg (2003)

[Pas06] Pass, R.: Parallel repetition of zero-knowledge proofs and the possibility of basing cryptography on np-hardness. In: IEEE Conference on Computational Complexity, pp. 96-110 (2006)

[Pas11] Pass, R.: Limits of provable security from standard assumptions. In: STOC, pp. 109-118 (2011)

[PPV08] Pandey, O., Pass, R., Vaikuntanathan, V.: Adaptive One-Way Functions and Applications. In: Wagner, D. (ed.) CRYPTO 2008. LNCS, vol. 5157, pp. 57-74. Springer, Heidelberg (2008)

[PR05a] Pass, R., Rosen, A.: Concurrent non-malleable commitments. In: FOCS 2005, pp. 563-572 (2005)

[PR05b] Pass, R., Rosen, A.: New and improved constructions of non-malleable cryptographic protocols. In: STOC 2005, pp. 533-542 (2005)

[PTV11] Pass, R., Tseng, W.-L.D., Venkitasubramaniam, M.: Towards Non-BlackBox Lower Bounds in Cryptography. In: Ishai, Y. (ed.) TCC 2011. LNCS, vol. 6597, pp. 579-596. Springer, Heidelberg (2011)

[PW10] Pass, R., Wee, H.: Constant-Round Non-malleable Commitments from Sub-exponential One-Way Functions. In: Gilbert, H. (ed.) EUROCRYPT 2010. LNCS, vol. 6110, pp. 638-655. Springer, Heidelberg (2010)

[RTV04] Reingold, O., Trevisan, L., Vadhan, S.P.: Notions of Reducibility between Cryptographic Primitives. In: Naor, M. (ed.) TCC 2004. LNCS, vol. 2951, pp. 1-20. Springer, Heidelberg (2004)

[RV10] Rothblum, G.N., Vadhan, S.P.: Are pcps inherent in efficient arguments? Computational Complexity 19(2), 265-304 (2010)

[Wee10] Wee, H.: Black-box, round-efficient secure computation via nonmalleability amplification. In: FOCS 2010, pp. 531-540 (2010) 